\title{
Coherent versus incoherent dynamics in InAs quantum-dot active wave guides
}

Borri, Paola; Langbein, W.; Hvam, Jørn Märcher; Heinrichsdorff, F.; Mao, M.-H.; Bimberg, D.

Published in:

Journal of Applied Physics

Link to article, DOI:

$10.1063 / 1.1367410$

Publication date:

2001

Document Version

Publisher's PDF, also known as Version of record

Link back to DTU Orbit

Citation (APA):

Borri, P., Langbein, W., Hvam, J. M., Heinrichsdorff, F., Mao, M-H., \& Bimberg, D. (2001). Coherent versus incoherent dynamics in InAs quantum-dot active wave guides. Journal of Applied Physics, 89(11), 6542-6544. https://doi.org/10.1063/1.1367410

\section{General rights}

Copyright and moral rights for the publications made accessible in the public portal are retained by the authors and/or other copyright owners and it is a condition of accessing publications that users recognise and abide by the legal requirements associated with these rights.

- Users may download and print one copy of any publication from the public portal for the purpose of private study or research.

- You may not further distribute the material or use it for any profit-making activity or commercial gain

- You may freely distribute the URL identifying the publication in the public portal 


\title{
Coherent versus incoherent dynamics in InAs quantum-dot active wave guides
}

\author{
P. Borria) and W. Langbein \\ Fachbereich Physik, Universität Dortmund, Otto-Hahn Strasse 4, 44227 Dortmund, Germany \\ J. M. Hvam \\ Research Center COM, The Technical University of Denmark, Building 349, DK-2800 Lyngby, Denmark \\ F. Heinrichsdorff, M.-H. Mao, and D. Bimberg \\ Institut für Festkörperphysik, Technische Universität Berlin, Hardenbergstr. 36, 10623 Berlin, Germany
}

(Received 11 July 2000; accepted for publication 26 February 2001)

\begin{abstract}
Coherent dynamics measured by time-resolved four-wave mixing is compared to incoherent population dynamics measured by differential transmission spectroscopy on the ground-state transition at room temperature of two types of InAs-based quantum dots with different confinement energies. The measurements are performed with heterodyne detection on quantum-dot active wave guides to enhance the light-matter interaction length. An elastic nature of the measured dephasing is revealed which is independent of the dot energy level scheme. (C) 2001 American Institute of Physics. [DOI: 10.1063/1.1367410]
\end{abstract}

Ultrafast carrier dynamics in self-organized InAs-based quantum dots (QDs) have been intensively investigated in recent years. ${ }^{7}$ The quasithree-dimensional confinement that occurs in semiconductor quantum dots strongly affects carrier radiative lifetimes as well as carrier-phonon and carriercarrier scattering rates. Intriguing effects such as long radiative lifetimes of several nanoseconds, inhibited interactions with optical and acoustic phonons in dots of small sizes from energy, and momentum conservation arguments, carriercarrier scattering of Auger type, have been widely debated in theory and experiments. For the application of QDs in optoelectronics, a key issue is the atomic-like density of states with sharp absorption lines, that follows from the lack of efficient carrier recombination and scattering mechanisms. ${ }^{1}$ Experimentally, ultranarrow homogeneous linewidths (few tens of $\mu \mathrm{eV}$ ) have been measured at low temperatures when isolating single $\mathrm{In}(\mathrm{Ga}) \mathrm{As}$ QDs from an inhomogeneously broadened ensemble of dots having different sizes and/or In content. ${ }^{2}$ Therefore, the inhomogeneous broadening was for a long time considered the main limit in the achievement of a delta-like density of states in QDs. Recently, room temperature measurements of the homogeneous linewidth of In(Ga)As QDs have revealed a large homogeneous broadening in the range of several $\mathrm{meV}^{3,4}$ up to tens of $\mathrm{meV}$ in the presence of electrically injected carriers, ${ }^{5}$ comparable to presently achieved inhomogeneous broadenings. Thus, a different picture appears where QDs at room temperatures are significantly broadened by intrinsic mechanisms. This, on the one hand, reduces the expected superior performances based on a delta-like density of states in real QD lasers, on the other hand, it implies a different behavior in the laser action ${ }^{6}$ where all the spectral gain participate to lasing, important for cw high-power applications. In view of this scenario, a better understanding of the processes associated with the observed

a)Electronic mail: borri@fred.physik.uni-dortmund.de homogeneous broadening in QDs at room temperature is crucial for further development of QD-based devices.

The possibility to optically explore the ultrafast dynamics in InAs-based QDs, especially in the coherent regime by nonlinear experiments such as four-wave mixing (FWM), is limited by their small interaction volume. We have recently demonstrated that time-resolved FWM (TRFWM) in selforganized InAs QDs can be measured even at room temperature, by using optical wave guiding to increase the lightmatter interaction length. The coherent dynamics in a FWM experiment allow us to measure the polarization decay time (usually called dephasing time) which is inversely proportional to the homogeneous linewidth of an optical transition.

In this work we compare the coherent dynamics measured by time-resolved FWM with the incoherent population dynamics measured by differential transmission in two types of InAs-based QD active wave guides at room temperature. One structure consists of binary/ternary InAs/InGaAs QDs with a separation between the ground state-dot transition and the wetting layer of $\sim 110 \mathrm{meV}$. It is the same structure investigated in Refs. 3 and 5, showing a ground state dot transition at $1.08 \mu \mathrm{m}$ with a broadening of about $60 \mathrm{meV}$ from photoluminescence measurements. The second type consists of pure InAs quantum dots showing about $220 \mathrm{meV}$ separation between the dot ground state and the wetting layer. A ground-state dot transition at $1.1 \mu \mathrm{m}$ and a broadening of $\sim 90 \mathrm{meV}$ was measured by photoluminescence experiments. ${ }^{7}$ Both investigated samples are active ridge wave guides, as schematically shown in Fig. 1, grown by metalorganic chemical vapor deposition in a positiveintrinsic-negative diode structure. Two $\mathrm{Al}_{0.7} \mathrm{Ga}_{0.3}$ As cladding layers and a ridge structure of $8 \mu \mathrm{m}$ width and $\sim 500 \mu \mathrm{m}$ length provide optical confinement and wave guiding. ${ }^{6}$ The active region consists of three stacked layers of QDs separated by $21 \mathrm{~nm}$ thick GaAs barriers and placed in the center of a $120 \mathrm{~nm} \mathrm{GaAs}$ layer. Electrical injection allowed laser 

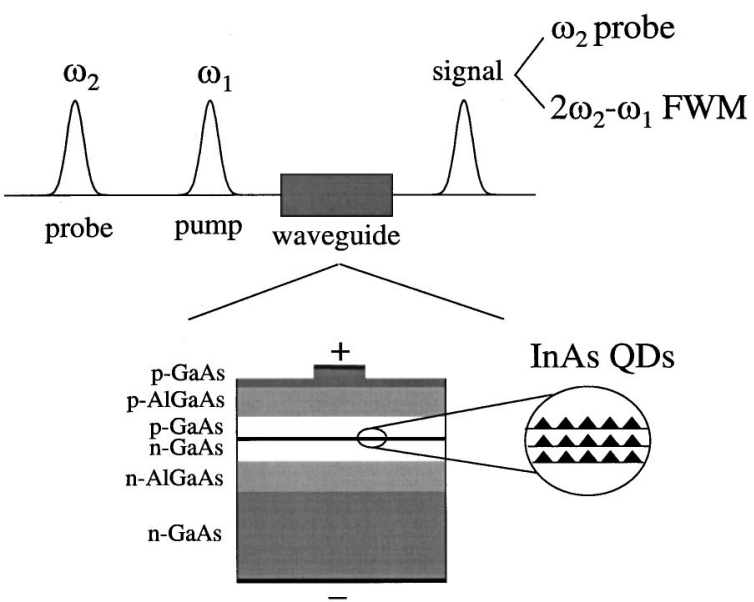

FIG. 1. Sketch of the experimental heterodyne setup and of the QD wave guide structure. $\omega_{1}$ and $\omega_{2}$ are radio-frequency shifts to the optical laser frequency in a quasidegenerate scheme. The signal is detected at the probe frequency for differential transmission spectroscopy or at the four-wave mixing frequency for coherent spectroscopy.

action, ${ }^{8,5}$ however in this work we concentrate on the QD absorption dynamics without bias current. The end facets of the wave guides were tilted, avoiding backreflection into the wave guide mode.

We performed heterodyne pump-probe and FWM experiments as schematically shown in Fig. 1. In this experiment laser pulses are provided by the idler wave of an optical parametric amplifier pumped by a Ti:Sa regenerative amplifier at $300 \mathrm{kHz}$ repetition rate. Fourier-limited $150 \mathrm{fs}$ pulses with $14 \mathrm{meV}$ spectral width centered at the dot-ground state transition wavelength were used. Two copropagating and copolarized pulses (in the transverse electric wave guide mode) were coupled in and out of the wave guide by high numerical aperture lenses (NA 0.65). In the differential transmission experiment, the transmitted probe was distinguished by the pump pulse using an heterodyne detection scheme that we have recently demonstrated with a low repetition rate laser system. ${ }^{9}$ The basic principle consists of a radio-frequency shift of the optical laser frequencies of pump and probe pulses and in a frequency selection of the transmitted probe. The same concept is applied in the FWM experiment where the signal is detected at the mixed frequency $2 \omega_{2}-\omega_{1}$, with $\omega_{2}$ and $\omega_{1}$ radio frequency shifts of the probe and pump pulses, respectively. ${ }^{3,5}$

The measured differential transmission at the dotground state transition is shown in Fig. 2 for the two investigated samples. Positive pump-probe delay times correspond to a pump pulse leading the probe. In the absorption case the dots are initially empty. When the pump arrives, bound electron-hole pairs (excitons) are created in the dot ground state, leading to a bleaching of the absorption (see sketch in figure). We estimated an absorption of the ground state transition from single pulse transmission experiments of $-3.35 \mathrm{~dB}$ for the InAs/InGaAs QD wave guide ${ }^{10}$ and of $-2.1 \mathrm{~dB}$ for the InAs QD waveguide, after correcting for coupling, wave guide, and reflection losses. Pump input energies of $\leqslant 0.5 \mathrm{pJ}$ per pulse resulted in an absorption bleaching of the ground state transition, and thus in an increased

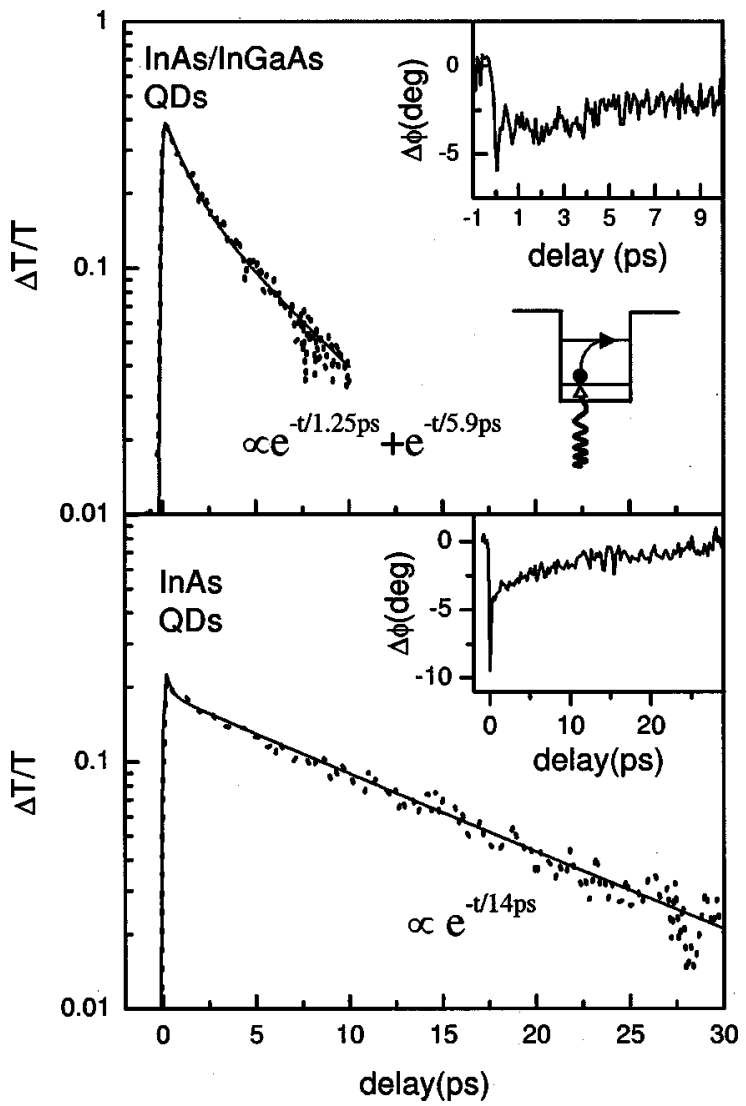

FIG. 2. Differential transmission at the ground state-dot transition measured in the two types of QDs, as indicated. Solid lines are fits to the data using the indicated response functions. A sketch of the absorption bleaching induced by the pump photons (curly arrow) that recover via carrier escape is also shown. In the insets, the probe phase dynamics measured in the heterodyne experiment are shown.

transmission, as shown in Fig. 2. The bleaching recovers by spontaneous recombination of the optically created carriers or by carrier escape from the dot ground state. Typical radiative lifetimes in QDs are in the nanosecond range, ${ }^{11}$ much longer than the measured absorption bleaching recovery, which is then attributed to carrier escape. We measured the same dynamics when decreasing the pump input energy, or when applying a small forward bias current such that a flat band configuration is realized in the active region of the diode. Thus, Auger type of escape and carrier tunneling is unlikely to play a role and we attribute the carrier escape to thermal activation via phonon absorption. Note that the lack of a long-lived population in the differential transmission, which one would expect once the thermal equilibrium is reached, is due to the diode structure of the sample. Once the carriers have escaped from the ground state, the built-in electric field of the diode at zero bias removes them out of the active region. When a flat band configuration is realized by a small applied bias current, long-lived population effects are observed.

In Fig. 2 a fit to the data is obtained by convoluting the pulse intensity autocorrelation with a response function. ${ }^{10}$ We find a good fit of the dynamics in the InAs/InGaAs QDs using a biexponential response function with two equally weighted time constants of $1.25 \pm 0.03$ ps and $5.9 \pm 0.1$ ps. $^{10}$ 


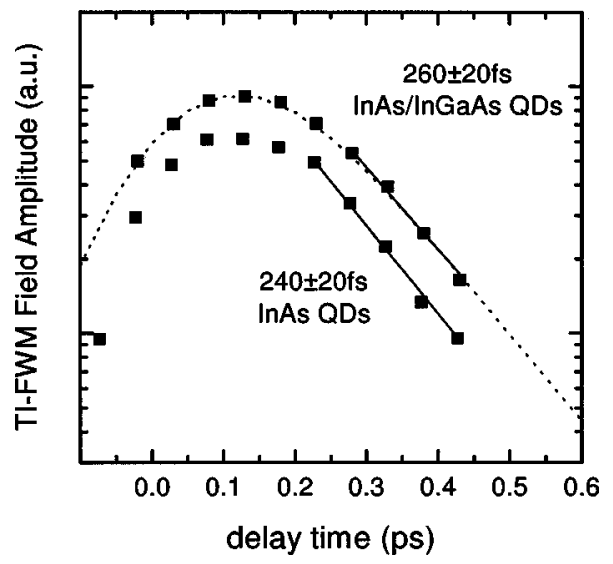

FIG. 3. Time-integrate FWM vs relative delay time of the exciting pulses in the two types of QDs. The dotted line is a numerical calculation, while the solid lines are exponential fits of the dynamics at positive delay. The inferred dephasing times are indicated.

They can be attributed to hole and electron escape times, since the absorption bleaching is proportional to the sum of electron and hole occupation numbers. The escape, which represents a heating of the optically excited excitons, also appears in the probe phase dynamics which is detected in an heterodyne experiment ${ }^{9}$ (see inset of Fig. 2). The differential transmission dynamics in the InAs QDs is much slower than what is measured for the InAs/InGaAs QDs, consistent with a larger confinement energy in the InAs QDs. The dynamics is dominated by a monoexponential decay of $14 \pm 0.3$ ps time constant. A initial faster transient of $\sim 0.3$ ps also appears but with smaller weight than the long one. Thus, a simple model of electron and hole escape with equally weighted time constants does not apply in this case, possibly due to the interplay between carrier escape and relaxation back from the excited states which complicates the overall escape dynamics in these strongly confined QDs. A carrier heating associated with the escape is also observed in the phase dynamics for these QDs.

In order to compare the incoherent population dynamics of the ground state-dot transition with the coherent dynamics of the polarization decay we have performed timeresolved FWM measurements. TRFWM of the dot ground state in the InAs/InGaAs active wave guide is described in detail in Ref. 3. The time integrated data are shown in Fig. 3 versus the relative delay time between the exciting pulses. The dotted lines are numerical calculation as described in Ref. 3 from which a dephasing time of $260 \pm 20$ ps was inferred, in agreement with the one deduced by the exponential decay of the FWM at long positive delay times. The shown measurements are in the low excitation regime $(\sim 0.1 \mathrm{pJ}$ energy per pulse) where the FWM signal followed a third-order behavior. ${ }^{3}$ Similarly, we have performed TRFWM in the InAs QD wave guide. The data are shown in Fig. 3 in the same low excitation regime, also within a third-order behavior for this sample. The dephasing time deduced from the
FWM exponential decay at long positive delay times is indicated. It can be clearly seen that the FWM dynamics in the two QD samples are very similar. Note that $250 \mathrm{fs}$ dephasing time corresponds to $5.3 \mathrm{meV}$ homogeneous broadening. Thus, both samples show fast coherent dynamics, corresponding to a homogeneous broadening at room temperature of several $\mathrm{meV}$, independent of the different confinement energies in the two dot structures. Moreover, the measured dephasing time is much faster than the incoherent population lifetime, indicating the elastic nature of the dominant dephasing mechanism. Since the FWM was measured in the low excitation regime and without electrical injection, phonon scattering should be the dominant dephasing mechanism. It has been recently proposed theoretically that second-order interactions with longitudinal optical (LO) phonons at room temperature can lead to efficient elastic dephasing without requiring that the energy level separation in the dots matches the LO phonon energy. ${ }^{12}$

In conclusion, pronounced differences in the population dynamics of the dot-ground state transition, due to carrier thermal escape in the range of several picosecond, are measured at room temperature in two types of InAs-based QDs with different confinement energies. In contrast, similar coherent dynamics are found, corresponding to a homogeneous broadening of several $\mathrm{meV}$. The elastic nature of the dephasing, attributed to phonon interactions, is evidenced by this comparison. Moreover, these results indicate that the homogeneous broadening of InAs-based QDs at room temperature is independent of their confinement energy. This aspect is very important in future development of QD-based devices.

This work was supported by the Danish Technical Research Council in the framework of SCOOP and by DFG in the framework of SFB 296. The authors acknowledge Tele Danmark R and D for the donation of part of the equipment.

${ }^{1}$ D. Bimberg, M. Grundmann, and N. N. Ledentsov, Quantum Dot Heterostructures (Wiley, Chichester, 1998).

${ }^{2}$ H. Kamada, J. Temmyo, M. Notomi, T. Furuta, and T. Tamamura, Jpn. J. Appl. Phys., Part 1 36, 4194 (1997).

${ }^{3}$ P. Borri, W. Langbein, J. Mørk, J. M. Hvam, F. Heinrichsdorff, M.-H. Mao, and D. Bimberg, Phys. Rev. B 60, 7784 (1999).

${ }^{4}$ K. Matsuda, T. Saiki, H. Saito, and K. Nishi, Appl. Phys. Lett. 76, 73 (2000)

${ }^{5}$ P. Borri, W. Langbein, J. M. Hvam, F. Heinrichsdorff, M.-H. Mao, and D. Bimberg, Appl. Phys. Lett. 76, 1380 (2000).

${ }^{6}$ M. Sugawara, K. Mukai, Y. Nakata, H. Ishikawa, and A. Sakamoto, Phys. Rev. B 61, 7595 (2000).

${ }^{7}$ F. Heinrichsdorff, Ph.D. thesis, Institut für Festkörperphysik, Technische Universität Berlin, 1997.

${ }^{8}$ F. Heinrichsdorff, M.-H. Mao, N. Kirstaedter, A. Krost, D. Bimberg, A. O. Kosogov, and P. Werner, Appl. Phys. Lett. 71, 22 (1997).

${ }^{9}$ P. Borri, W. Langbein, J. Mork, and J. M. Hvam, Opt. Commun. 169, 317 (1999).

${ }^{10}$ P. Borri, W. Langbein, J. M. Hvam, F. Heinrichsdorff, M.-H. Mao, and D. Bimberg, IEEE Photonics Technol. Lett. 12, 594 (2000); IEEE J. Sel. Top. Quantum Electron. 6, 544 (2000).

${ }^{11}$ W. Yang, R. R. Lowe-Webb, H. Lee, and P. C. Sercel, Phys. Rev. B 56, 13314 (1997).

${ }^{12}$ A. V. Uskov, B. Tromborg, A. P. Jauho, J. Mbrk, and R. Lang, Phys. Rev. Lett. 85, 1516 (2000). 